\title{
Weak convergence theorem for the three-step iterations of non-Lipschitzian nonself mappings in Banach spaces
}

\author{
Lanping Zhu, Qianglian Huang ${ }^{*}$ and Xiaoru Chen
}

\author{
* Correspondence: \\ qlhmath@yahoo.com.cn \\ College of Mathematics, Yangzhou \\ University, Yangzhou 225002, China
}

\begin{abstract}
In this article, we introduce a new three-step iterative scheme for the mappings which are asymptotically nonexpansive in the intermediate sense in Banach spaces. Weak convergence theorem is established for this three-step iterative scheme in a uniformly convex Banach space that satisfies Opial's condition or whose dual space has the Kadec-Klee property. Furthermore, we give an example of the nonself mapping which is asymptotically nonexpansive in the intermediate sense but not asymptotically nonexpansive. The results obtained in this article extend and improve many recent results in this area.
\end{abstract}

AMS classification: 47H10; 47H09; 46B2O.

Keywords: asymptotically nonexpansive in the intermediate sense non-self mapping, Kadec-Klee property, Opial?'?s condition, common fixed point

\section{Introduction}

Fixed-point iterations process for nonexpansive and asymptotically nonexpansive mappings in Banach spaces have been studied extensively by various authors [1-13]. In 1991, Schu [4] considered the following modified Mann iteration process for an asymptotically nonexpansive map $T$ on $C$ and a sequence $\left\{\alpha_{n}\right\}$ in $[0,1]$ :

$$
x_{1} \in C, \quad x_{n+1}=\alpha_{n} x_{n}+\left(1-\alpha_{n}\right) T^{n} x_{n}, \quad n \geq 1 .
$$

Since then, Schu's iteration process (1.1) has been widely used to approximate fixed points of asymptotically nonexpansive mappings in Hilbert spaces or Banach spaces [7,8,10-13]. Noor, in 2000, introduced a three-step iterative scheme and studied the approximate solutions of variational inclusion in Hilbert spaces [6]. Later, $\mathrm{Xu}$ and Noor [7], Cho et al. [8], Suantai [9], Plubtieng et al. [12] studied the convergence of the three-step iterations for asymptotically nonexpansive mappings in a uniformly convex Banach space which satisfies Opial's condition or whose norm is Fréchet differentiable.

In most of these articles, the operator $T$ remains a self-mapping of a nonempty closed convex subset $C$ of a uniformly convex Banach space $X$. If, however, the domain of $T, D(T)$, is a proper subset of $X$ (and this is the case in several applications), and $T$ maps $D(T)$ into $X$, then the iterative sequence $\left\{x_{n}\right\}$ may fail to be well defined. One method that has been used to overcome this is to introduce a retraction $P$. A subset $C$

(c) 2011 Zhu et al; licensee Springer. This is an Open Access article distributed under the terms of the Creative Commons Attribution License (http://creativecommons.org/licenses/by/2.0), which permits unrestricted use, distribution, and reproduction in any medium, provided the original work is properly cited. 
of $X$ is said to be retract if there exists continuous mapping $P: X \rightarrow C$ such that $P x=$ $x$ for all $x \in C$ and $P$ is said to be a retraction. Recent results on approximation of fixed points of nonexpansive or asymptotically nonexpansive nonself mappings can be found in [14-19] and the references cited therein. For example, in 2003, Chidume et al. [16] introduced the following modified Mann iteration process and got the convergence theorems for asymptotically nonexpansive nonself-mapping:

$$
x_{1} \in C, \quad x_{n+1}=P\left[\alpha_{n} x_{n}+\left(1-\alpha_{n}\right) T(P T)^{n-1} x_{n}\right], \quad n \geq 1 .
$$

Recently, Thianwan [18] generalized the iteration process (1.2) as follows: $x_{1} \in C$,

$$
\begin{aligned}
x_{n+1} & =P\left[\alpha_{n} y_{n}+\left(1-\alpha_{n}\right) T_{1}\left(P T_{1}\right)^{n-1} y_{n}\right] ; \\
y_{n} & =P\left[\beta_{n} x_{n}+\left(1-\beta_{n}\right) T_{2}\left(P T_{2}\right)^{n-1} x_{n}\right] .
\end{aligned}
$$

Obviously, if $\beta_{n}=1$ for all $n \geq 1$, then (1.3) reduces to (1.2). Thianwan [18] proved the weak convergence theorem of the iteration process (1.3) in uniformly convex Banach spaces that satisfy Opial's condition.

The concept of asymptotically nonexpansive in the intermediate sense nonself mappings was introduced by Chidume et al. [20] as an important generalization of asymptotically nonexpansive in the intermediate sense self-mappings.

Definition 1.1 Let $C$ be a nonempty subset of a Banach space $X$. Let $P: X \rightarrow C$ be a nonexpansive retraction of $X$ onto $C$. A nonself mapping $T: C \rightarrow X$ is called asymptotically nonexpansive in the intermediate sense if $T$ is continuous and the following inequality holds:

$$
\limsup _{n \rightarrow+\infty} \sup _{x, y \in C}\left(\left\|T(P T)^{n-1} x-T(P T)^{n-1} y\right\|-\|x-y\|\right) \leq 0 .
$$

It should be noted that in [20-22], the asymptotically nonexpansive in the intermediate sense mapping is required to be uniformly continuous. In this article, we assume the continuity of $T$ instead of uniform continuity. Chidume et al. [20] gave the weak convergence theorem for uniformly continuous nonself mapping which is asymptotically nonexpansive in the intermediate sense in uniformly convex Banach space whose dual space has the Kadec-Klee property.

Inspired and motivated by $[16,18,20]$, we investigate the weak convergence theorem of three-step iteration process for continuous nonself mappings which are asymptotically nonexpansive in the intermediate sense in this article. Since the asymptotically nonexpansive in the intermediate sense mappings are non-Lipschitzian and Bruck's Lemma [23] do not extend beyond Lipschitzian mappings, new techniques are needed for this more general case. Utilizing the technique of the modulus of convexity and a new demiclosed principle for nonself-maps of Kazor [24], we establish the weak convergence theorem of the three-step iterative scheme in a uniformly convex Banach space that satisfies Opial's condition or whose dual space has the Kadec-Klee property, which extends and improves the recently announced ones in $[4,16,18-20]$. It should be noted that our theorems are new even in the case that the space has a Fréchet differentiable norm. In the end, to illustrate our theorem, we give a nonself mapping which is asymptotically nonexpansive in the intermediate sense but not asymptotically nonexpansive. 


\section{Preliminaries}

Let $X$ be a Banach space and $X^{*}$ be its dual, then the value of $x^{*} \in X^{*}$ at $x \in X$ will be denoted by $\left\langle x, x^{*}\right\rangle$ and we associate the set

$$
J(x)=\left\{x^{*} \in X^{*}:\left\langle x, x^{*}\right\rangle=\|x\|^{2}=\left\|x^{*}\right\|^{2}\right\} .
$$

It follows from the Hahn-Banach theorem that $J(x) \neq \varnothing$ for any $x \in X$. Then the multi-valued operator $J: X \mapsto X^{*}$ is called the normalized duality mapping of $X$. Recall that a Banach space $X$ is said to be uniformly convex if for each $\varepsilon \in[0,2]$, the modulus of convexity of $X$ defined by

$$
\delta(\varepsilon)=\inf \left\{1-\frac{1}{2}\|x+y\|:\|x\| \leq 1,\|y\| \leq 1,\|x-y\| \geq \varepsilon\right\},
$$

satisfies the inequality $\delta(\varepsilon)>0$ for all $\varepsilon>0$. Note that every closed convex subset of a uniformly convex Banach space is a retract. We say that $X$ has the Kadec-Klee property if for every sequence $\left\{x_{n}\right\} \subset X$, whenever $x_{n} \rightarrow x$ with $\left\|x_{n}\right\| \rightarrow\|x\|$, it follows that $x_{n} \rightarrow x$. We would like to remark that a reflexive Banach space $X$ with a Fréchet differentiable norm implies that its dual $X^{*}$ has Kadec-Klee property, while the converse implication fails [25].

Recall that a Banach space $X$ is said to satisfies Opial's condition if $x_{n}-x$ and $x \neq y$ implies that

$$
\limsup _{n \rightarrow+\infty}\left\|x_{n}-x\right\|<\limsup _{n \rightarrow+\infty}\left\|x_{n}-y\right\| .
$$

The following lemmas are needed to prove our main results in next section.

Lemma 2.1 [5]Let the nonnegative number sequences $\left\{c_{n}\right\}$ and $\left\{w_{n}\right\}$ satisfy

$$
c_{n+1} \leq c_{n}+w_{n}, \quad n \in N
$$

If $\sum_{n=1}^{+\infty} w_{n}<+\infty$, then $\lim _{n \rightarrow+\infty} c_{n e x i s t s .}$

Lemma 2.2 [4] Suppose that $X$ is a uniformly convex Banach space and for all positive integers $n, 0<p \leq t_{n} \leq q<1$. If $\left\{x_{n}\right\}$ and $\left\{y_{n}\right\}$ are two sequences of $X$ such that $\limsup _{n \rightarrow+\infty}\left\|y_{n}\right\| \leq r, \limsup _{n \rightarrow+\infty}\left\|y_{n}\right\| \leq r_{\text {and }}$

$$
\lim _{n \rightarrow+\infty}\left\|t_{n} x_{n}+\left(1-t_{n}\right) y_{n}\right\|=r
$$

hold for some $r \geq 0$. Then $\lim _{n \rightarrow+\infty}\left\|x_{n}-y_{n}\right\|=0$.

Lemma 2.3 [3] Let $X$ be a uniformly convex Banach space. If $\|x\| \leq 1,\|y\| \leq 1$ and $\| x-y|| \geq \varepsilon>0$, then for all $\lambda \in[0,1]$,

$$
\|\lambda x+(1-\lambda) y\| \leq 1-2 \lambda(1-\lambda) \delta(\varepsilon) .
$$

Lemma 2.4 [26] Let $X$ be a Banach space and $J$ be the normalized duality mapping. Then for given $x, y \in X$ and $j(x+y) \in J(x+y)$, we have

$$
\|x+y\|^{2} \leq\|x\|^{2}+2\langle y, j(x+y)\rangle .
$$

Lemma 2.5 (Demiclosed principle for nonself-map [24]) Let $C$ be a nonempty closed convex subset of a uniformly convex Banach space $X$ and $T: C \rightarrow X$ be a nonself mapping which is continuous and asymptotically nonexpansive in the intermediate sense. If $\left\{x_{n}\right\}$ is a sequence in $C$ converging weakly to $x$ and 


$$
\lim _{k \rightarrow+\infty} \limsup _{n \rightarrow+\infty}\left\|x_{n}-T(P T)^{k-1} x_{n}\right\|=0,
$$

then $x \in F(T)$, i.e., $T x=x$.

\section{Main results}

Let $C$ be a nonempty closed convex subset of a uniformly convex Banach space $X$ and $P: X \rightarrow C$ be a nonexpansive retraction from $X$ onto $C$. Let $T_{1}, T_{2}, T_{3}: C \rightarrow X$ be three continuous nonself mappings which are asymptotically nonexpansive in the intermediate sense. Suppose that

$$
r_{n}=\max \left\{0, \sup _{x, y \in C ; i=1,2,3 .}\left\|T_{i}\left(P T_{i}\right)^{n-1} x-T_{i}\left(P T_{i}\right)^{n-1} y\right\|-\|x-y\|\right\},
$$

then $r_{n} \geq 0, \lim _{n \rightarrow+\infty} r_{n}=0$ and for all $x, y \in C$ and $n \in N$,

$$
\left\|T_{i}\left(P T_{i}\right)^{n-1} x-T_{i}\left(P T_{i}\right)^{n-1} y\right\|-\|x-y\| \leq r_{n}, \quad i=1,2,3 .
$$

For a given $x_{1} \in C$, we define the sequence $\left\{x_{n}\right\} \subset C$ by

$$
\begin{aligned}
x_{n+1} & =P\left[\alpha_{n}^{(1)} z_{n}+\left(1-\alpha_{n}^{(1)}\right) T_{1}\left(P T_{1}\right)^{n-1} z_{n}\right] ; \\
z_{n} & =P\left[\alpha_{n}^{(2)} y_{n}+\left(1-\alpha_{n}^{(2)}\right) T_{2}\left(P T_{2}\right)^{n-1} y_{n}\right] ; \\
y_{n} & =P\left[\alpha_{n}^{(3)} x_{n}+\left(1-\alpha_{n}^{(3)}\right) T_{3}\left(P T_{3}\right)^{n-1} x_{n}\right] .
\end{aligned}
$$

where $\left\{\alpha_{n}^{(i)}\right\}$ is in $0[1]$ with $0<p \leq \alpha_{n}^{(i)} \leq q<1, i=1,2,3$.

We also assume that the sequence $\left\{r_{n}\right\}$ satisfies $\sum_{n=1}^{+\infty} r_{n}<+\infty$ and the set of common fixed points of $\left\{T_{i}\right\}_{i=1}^{3}$ is nonempty, i.e.,

$$
F=\cap_{i=1}^{3} F\left(T_{i}\right)=\left\{x \in C: T_{1} x=T_{2} x=T_{3} x=x\right\} \neq \emptyset .
$$

\section{Lemma 3.1}

$$
\lim _{n \rightarrow+\infty}\left\|x_{n}-f\right\|=\lim _{n \rightarrow+\infty}\left\|y_{n}-f\right\|=\lim _{n \rightarrow+\infty}\left\|z_{n}-f\right\|=r
$$

exists for all $f \in F$.

Proof. For all $f \in F$,

$$
\begin{aligned}
& \left\|y_{n}-f\right\|=\left\|P\left[\alpha_{n}^{(3)} x_{n}+\left(1-\alpha_{n}^{(3)}\right) T_{3}\left(P T_{3}\right)^{n-1} x_{n}\right]-f\right\| \\
\leq & \left\|\left[\alpha_{n}^{(3)} x_{n}+\left(1-\alpha_{n}^{(3)}\right) T_{3}\left(P T_{3}\right)^{n-1} x_{n}\right]-f\right\| \\
\leq & \alpha_{n}^{(3)}\left\|x_{n}-f\right\|+\left(1-\alpha_{n}^{(3)}\right)\left\|T_{3}\left(P T_{3}\right)^{n-1} x_{n}-f\right\| \\
= & \left\|x_{n}-f\right\|+r_{n} .
\end{aligned}
$$

Hence

$$
\begin{aligned}
& \left\|z_{n}-f\right\|=\left\|P\left[\alpha_{n}^{(2)} y_{n}+\left(1-\alpha_{n}^{(2)}\right) T_{2}\left(P T_{2}\right)^{n-1} y_{n}\right]-f\right\| \\
\leq & \left\|\left[\alpha_{n}^{(2)} y_{n}+\left(1-\alpha_{n}^{(2)}\right) T_{2}\left(P T_{2}\right)^{n-1} y_{n}\right]-f\right\| \\
\leq & \left\|y_{n}-f\right\|+r_{n} \\
\leq & \left\|x_{n}-f\right\|+2 r_{n} .
\end{aligned}
$$


Thus

$$
\begin{aligned}
& \left\|x_{n+1}-f\right\|=\left\|P\left[\alpha_{n}^{(1)} z_{n}+\left(1-\alpha_{n}^{(1)}\right) T_{1}\left(P T_{1}\right)^{n-1} z_{n}\right]-f\right\| \\
\leq & \left\|\left[\alpha_{n}^{(1)} z_{n}+\left(1-\alpha_{n}^{(1)}\right) T_{1}\left(P T_{1}\right)^{n-1} z_{n}\right]-f\right\| \\
\leq & \left\|z_{n}-f\right\|+r_{n} \\
\leq & \left\|x_{n}-f\right\|+3 r_{n} .
\end{aligned}
$$

Put $w_{n}=3 r_{n}$, then we can obtain $\sum_{n=1}^{+\infty} w_{n}<+\infty$ and

$$
\left\|x_{n+1}-f\right\| \leq\left\|x_{n}-f\right\|+w_{n} .
$$

By Lemma 2.1, we can conclude that

$$
\lim _{n \rightarrow+\infty}\left\|x_{n}-f\right\|=r
$$

exists. Combining it with (3.4), we have

$$
\lim _{n \rightarrow+\infty}\left\|z_{n}-f\right\|=r .
$$

Hence by (3.3), we get

$$
\lim _{n \rightarrow+\infty}\left\|y_{n}-f\right\|=r \text {. }
$$

This completes the proof.

\section{Lemma 3.2}

$$
\lim _{k \rightarrow+\infty} \limsup _{n \rightarrow+\infty}\left\|x_{n}-T_{i}\left(P T_{i}\right)^{k-1} x_{n}\right\|=0, \quad i=1,2,3
$$

Proof. By (3.2) and (3.4), we can get

$$
\begin{aligned}
r & =\lim _{n \rightarrow+\infty}\left\|\left[\alpha_{n}^{(1)} z_{n}+\left(1-\alpha_{n}^{(1)}\right) T_{1}\left(P T_{1}\right)^{n-1} z_{n}\right]-f\right\| \\
& =\lim _{n \rightarrow+\infty}\left\|\left(1-\alpha_{n}^{(1)}\right)\left[T_{1}\left(P T_{1}\right)^{n-1} z_{n}-f\right]+\alpha_{n}^{(1)}\left(z_{n}-f\right)\right\|
\end{aligned}
$$

Then it follows from Lemma 2.2 and $\limsup _{n \rightarrow+\infty}\left\|T_{1}\left(P T_{1}\right)^{n-1} z_{n}-f\right\| \leq r$ that

$$
\lim _{n \rightarrow+\infty}\left\|T_{1}\left(P T_{1}\right)^{n-1} z_{n}-z_{n}\right\|=0
$$

According to (3.3), we have

$$
\begin{aligned}
r & =\lim _{n \rightarrow+\infty}\left\|\left[\alpha_{n}^{(2)} y_{n}+\left(1-\alpha_{n}^{(2)}\right) T_{2}\left(P T_{2}\right)^{n-1} y_{n}\right]-f\right\| \\
& =\lim _{n \rightarrow+\infty}\left\|\left(1-\alpha_{n}^{(2)}\right)\left[T_{2}\left(P T_{2}\right)^{n-1} y_{n}-f\right]+\alpha_{n}^{(2)}\left(y_{n}-f\right)\right\|
\end{aligned}
$$

Noting $\underset{n \rightarrow+\infty}{\limsup }\left\|T_{2}\left(P T_{2}\right)^{n-1} y_{n}-f\right\| \leq r$, by Lemma 2.2 again, we can get

$$
\lim _{n \rightarrow+\infty}\left\|T_{2}\left(P T_{2}\right)^{n-1} y_{n}-y_{n}\right\|=0
$$

Similarly, we can obtain

$$
\lim _{n \rightarrow+\infty}\left\|T_{3}\left(P T_{3}\right)^{n-1} x_{n}-x_{n}\right\|=0
$$


Hence, it follows from

$$
\begin{aligned}
\left\|y_{n}-x_{n}\right\| & =\left\|P\left[\alpha_{n}^{(3)} x_{n}+\left(1-\alpha_{n}^{(3)}\right) T_{3}\left(P T_{3}\right)^{n-1} x_{n}\right]-x_{n}\right\| \\
& \leq\left\|\left[\alpha_{n}^{(3)} x_{n}+\left(1-\alpha_{n}^{(3)}\right) T_{3}\left(P T_{3}\right)^{n-1} x_{n}\right]-x_{n}\right\| \\
& \leq\left\|T_{3}\left(P T_{3}\right)^{n-1} x_{n}-x_{n}\right\| .
\end{aligned}
$$

that $\lim _{n \rightarrow+\infty}\left\|y_{n}-x_{n}\right\|=0$. Also, we can see

$$
\begin{aligned}
\left\|z_{n}-y_{n}\right\| & =\left\|P\left[\alpha_{n}^{(2)} y_{n}+\left(1-\alpha_{n}^{(2)}\right) T_{2}\left(P T_{2}\right)^{n-1} y_{n}\right]-y_{n}\right\| \\
& \leq\left\|\left[\alpha_{n}^{(2)} y_{n}+\left(1-\alpha_{n}^{(2)}\right) T_{2}\left(P T_{2}\right)^{n-1} y_{n}\right]-y_{n}\right\| \\
& \leq\left\|T_{2}\left(P T_{2}\right)^{n-1} y_{n}-y_{n}\right\|
\end{aligned}
$$

and

$$
\begin{aligned}
\left\|x_{n+1}-z_{n}\right\| & =\left\|P\left[\alpha_{n}^{(1)} z_{n}+\left(1-\alpha_{n}^{(1)}\right) T_{1}\left(P T_{1}\right)^{n-1} z_{n}\right]-z_{n}\right\| \\
& \leq\left\|\left[\alpha_{n}^{(1)} z_{n}+\left(1-\alpha_{n}^{(1)}\right) T_{1}\left(P T_{1}\right)^{n-1} z_{n}\right]-z_{n}\right\| \\
& \leq\left\|T_{1}\left(P T_{1}\right)^{n-1} z_{n}-z_{n}\right\| .
\end{aligned}
$$

It follows from (3.5) and (3.6) that

$$
\lim _{n \rightarrow+\infty}\left\|x_{n+1}-z_{n}\right\|=\lim _{n \rightarrow+\infty}\left\|z_{n}-y_{n}\right\|=0 .
$$

Hence $\lim _{n \rightarrow+\infty}\left\|x_{n+1}-x_{n}\right\|=\lim _{n \rightarrow+\infty}\left\|z_{n}-x_{n}\right\|=0$. Thus for any fixed $k \in N$,

$$
\lim _{n \rightarrow+\infty}\left\|x_{n+k}-x_{n}\right\|=0 .
$$

Noting (3.7) and

$$
\begin{aligned}
& \left\|x_{n}-T_{3}\left(P T_{3}\right)^{k-1} x_{n}\right\| \\
\leq & \left\|x_{n}-x_{n+k}\right\|+\left\|x_{n+k}-T_{3}\left(P T_{3}\right)^{n+k-1} x_{n+k}\right\|+\| T_{3}\left(P T_{3}\right)^{n+k-1} x_{n+k} \\
& \quad-T_{3}\left(P T_{3}\right)^{n+k-1} x_{n}\|+\| T_{3}\left(P T_{3}\right)^{n+k-1} x_{n}-T_{3}\left(P T_{3}\right)^{k-1} x_{n} \| \\
\leq & \left\|x_{n}-x_{n+k}\right\|+\left\|x_{n+k}-T_{3}\left(P T_{3}\right)^{n+k-1} x_{n+k}\right\|+\left\|x_{n+k}-x_{n}\right\|+r_{n+k} \\
& +\left\|T_{3}\left(P T_{3}\right)^{n-1} x_{n}-x_{n}\right\|+r_{k}
\end{aligned}
$$

we have $\limsup _{n \rightarrow+\infty}\left\|x_{n}-T_{3}\left(P T_{3}\right)^{k-1} x_{n}\right\| \leq r_{k}$, which implies

$$
\lim _{k \rightarrow+\infty} \limsup _{n \rightarrow+\infty}\left\|x_{n}-T_{3}\left(P T_{3}\right)^{k-1} x_{n}\right\|=0 .
$$

Combining (3.6) with

$$
\begin{aligned}
& \left\|T_{2}\left(P T_{2}\right)^{n-1} x_{n}-x_{n}\right\| \\
\leq & \left\|T_{2}\left(P T_{2}\right)^{n-1} x_{n}-T_{2}\left(P T_{2}\right)^{n-1} y_{n}\right\|+\left\|T_{2}\left(P T_{2}\right)^{n-1} y_{n}-y_{n}\right\|+\left\|y_{n}-x_{n}\right\| \\
\leq & 2\left\|x_{n}-y_{n}\right\|+\left\|T_{2}\left(P T_{2}\right)^{n-1} y_{n}-y_{n}\right\|+r_{n}
\end{aligned}
$$

we can see $\lim _{n \rightarrow+\infty}\left\|T_{2}\left(P T_{2}\right)^{n-1} x_{n}-x_{n}\right\|=0$. Thus

$$
\begin{aligned}
& \left\|x_{n}-T_{2}\left(P T_{2}\right)^{k-1} x_{n}\right\| \\
\leq & \left\|x_{n}-x_{n+k}\right\|+\left\|x_{n+k}-T_{2}\left(P T_{2}\right)^{n+k-1} x_{n+k}\right\|+\| T_{2}\left(P T_{2}\right)^{n+k-1} x_{n+k} \\
& \quad-T_{2}\left(P T_{2}\right)^{n+k-1} x_{n}\|+\| T_{2}\left(P T_{2}\right)^{n+k-1} x_{n}-T_{2}\left(P T_{2}\right)^{k-1} x_{n} \| \\
\leq & \left\|x_{n}-x_{n+k}\right\|+\left\|x_{n+k}-T_{2}\left(P T_{2}\right)^{n+k-1} x_{n+k}\right\|+\left\|x_{n+k}-x_{n}\right\|+r_{n+k} \\
& +\left\|T_{2}\left(P T_{2}\right)^{n-1} x_{n}-x_{n}\right\|+r_{k}
\end{aligned}
$$


which implies

$$
\lim _{k \rightarrow+\infty} \limsup _{n \rightarrow+\infty}\left\|x_{n}-T_{2}\left(P T_{2}\right)^{k-1} x_{n}\right\|=0 .
$$

Combining (3.5) with

$$
\begin{aligned}
& \left\|T_{1}\left(P T_{1}\right)^{n-1} x_{n}-x_{n}\right\| \\
\leq & \left\|T_{1}\left(P T_{1}\right)^{n-1} x_{n}-T_{1}\left(P T_{1}\right)^{n-1} z_{n}\right\|+\left\|T_{1}\left(P T_{1}\right)^{n-1} z_{n}-z_{n}\right\|+\left\|x_{n}-x_{n}\right\| \\
\leq & 2\left\|x_{n}-z_{n}\right\|+\left\|T_{1}\left(P T_{1}\right)^{n-1} z_{n}-z_{n}\right\|+r_{n}
\end{aligned}
$$

we can see $\lim _{n \rightarrow+\infty}\left\|T_{1}\left(P T_{1}\right)^{n-1} x_{n}-x_{n}\right\|=0$. Thus

$$
\begin{aligned}
& \left\|x_{n}-T_{1}\left(P T_{1}\right)^{k-1} x_{n}\right\| \\
\leq & \left\|x_{n}-x_{n+k}\right\|+\left\|x_{n+k}-T_{1}\left(P T_{1}\right)^{n+k-1} x_{n+k}\right\|+\| T_{1}\left(P T_{1}\right)^{n+k-1} x_{n+k} \\
& -T_{1}\left(P T_{1}\right)^{n+k-1} x_{n}\|+\| T_{1}\left(P T_{1}\right)^{n+k-1} x_{n}-T_{1}\left(P T_{1}\right)^{k-1} x_{n} \| \\
\leq & \left\|x_{n}-x_{n+k}\right\|+\left\|x_{n+k}-T_{1}\left(P T_{1}\right)^{n+k-1} x_{n+k}\right\|+\left\|x_{n+k}-x_{n}\right\|+r_{n+k} \\
& +\left\|T_{1}\left(P T_{1}\right)^{n-1} x_{n}-x_{n}\right\|+r_{k}
\end{aligned}
$$

which implies

$$
\lim _{k \rightarrow+\infty} \limsup _{n \rightarrow+\infty}\left\|x_{n}-T_{1}\left(P T_{1}\right)^{k-1} x_{n}\right\|=0 .
$$

This completes the proof.

Define the operator $W_{n}: C \rightarrow C$ by

$$
\begin{aligned}
W_{n} x & =P\left[\alpha_{n}^{(1)} x^{(1)}+\left(1-\alpha_{n}^{(1)}\right) T_{1}\left(P T_{1}\right)^{n-1} x^{(1)}\right] ; \\
x^{(1)} & =P\left[\alpha_{n}^{(2)} x^{(2)}+\left(1-\alpha_{n}^{(2)}\right) T_{2}\left(P T_{2}\right)^{n-1} x^{(2)}\right] ; \\
x^{(2)} & =P\left[\alpha_{n}^{(3)} x+\left(1-\alpha_{n}^{(3)}\right) T_{3}\left(P T_{3}\right)^{n-1} x\right],
\end{aligned}
$$

where $x \in C$. Then by (3.1), $x_{n+1}=W_{n} x_{n}$ and for all $x, y \in C$, we have

$$
\begin{aligned}
\left\|x^{(2)}-y^{(2)}\right\| & \leq \alpha_{n}^{(3)}\|x-y\|+\left(1-\alpha_{n}^{(3)}\right)\left\|T_{3}\left(P T_{3}\right)^{n-1} x-T_{3}\left(P T_{3}\right)^{n-1} y\right\| \\
& \leq\|x-y\|+r_{n \prime} \\
\left\|x^{(1)}-y^{(1)}\right\| & \leq\left\|x^{(2)}-y^{(2)}\right\|+r_{n} \leq\|x-y\|+2 r_{n}
\end{aligned}
$$

and

$$
\left\|W_{n} x-W_{n} y\right\| \leq\|x-y\|+3 r_{n}=\|x-y\|+w_{n} .
$$

For any $f \in F$, we get $W_{n} f=f$. Set

$$
S_{n, m}=W_{n+m-1} W_{n+m-2} \cdots W_{n+1} W_{n}: C \rightarrow C,
$$

then $x_{n+m}=S_{n, m} x_{n}$ and for all $f \in F, S_{n, m} f=f$. Note that for any $x, y \in C$,

$$
\left\|S_{n, m} x-S_{n, m} y\right\| \leq\|x-y\|+\left(w_{n}+\cdots+w_{n+m-1}\right) .
$$

Lemma 3.3 Let $f, g \in F$ and $\lambda \in[0,1]$, then

$$
h(\lambda)=\lim _{n \rightarrow+\infty}\left\|\lambda x_{n}+(1-\lambda) f-g\right\|
$$

exists. 
Proof. It follows from Lemma 3.1 that $\lim _{n \rightarrow+\infty}\left\|x_{n}-f\right\|=r$ exists. If $\lambda=0,1$ or $r=0$, then the conclusion holds. Assume that $r>0$ and $\lambda \in(0,1)$, then for any $\varepsilon>0$, there exists $d>0(d<\varepsilon)$ such that

$$
(r+d)\left[1-2 \lambda(1-\lambda) \delta\left(\frac{\varepsilon}{r+d}\right)\right]<r-d,
$$

where $\delta(\cdot)$ is the modulus of convexity of the norm. Hence there exists a positive integer $n_{0}$ such that for all $n>n_{0}$,

$$
r-\frac{d}{4} \leq\left\|x_{n}-f\right\| \leq r+\frac{d}{4}
$$

and

$$
\sum_{i=n}^{+\infty} w_{i} \leq \lambda(1-\lambda) \frac{d}{4}<\frac{\varepsilon}{4}
$$

Now we claim that for all $n>n_{0}$,

$$
\left\|S_{n, m}\left[\lambda x_{n}+(1-\lambda) f\right]-\left[\lambda S_{n, m} x_{n}+(1-\lambda) f\right]\right\| \leq \varepsilon . \quad \forall m \in N
$$

Otherwise, we can suppose that there are some $n>n_{0}$ and some $m \in N$ such that

$$
\left\|S_{n, m}\left[\lambda x_{n}+(1-\lambda) f\right]-\left[\lambda S_{n, m} x_{n}+(1-\lambda) f\right]\right\| \geq \varepsilon .
$$

Put $z=\lambda x_{n}+(1-\lambda) f, x=(1-\lambda)\left(S_{n, m} z-f\right)$, and $y=\lambda\left(S_{n, m} x_{n}-S_{n, m} z\right)$, then by (3.8), (3.10), and (3.11), we have

$$
\begin{aligned}
\|x\| & =(1-\lambda)\left\|S_{n, m} z-f\right\| \\
& \leq(1-\lambda)\left[\|z-f\|+\left(w_{n+m-1}+\cdots+w_{n+1}+w_{n}\right)\right] \\
& \leq \lambda(1-\lambda)\left(\left\|x_{n}-f\right\|+\frac{d}{4}\right) \\
& \leq \lambda(1-\lambda)(r+d), \\
\|y\| & =\lambda\left\|S_{n, m} x_{n}-S_{n, m} z\right\| \\
& \leq \lambda\left[\left\|x_{n}-z\right\|+\left(w_{n+m-1}+\cdots+w_{n+1}+w_{n}\right)\right] \\
& \leq \lambda(1-\lambda)\left(\left\|x_{n}-f\right\|+\frac{d}{4}\right) \\
& \leq \lambda(1-\lambda)(r+d), \\
\|x-y\| & =\left\|S_{n, m}\left[\lambda x_{n}+(1-\lambda) f\right]-\left[\lambda S_{n, m} x_{n}+(1-\lambda) f\right]\right\| \geq \varepsilon
\end{aligned}
$$

and

$$
\lambda x+(1-\lambda) y=\lambda(1-\lambda)\left(S_{n, m} x_{n}-f\right) .
$$

So by Lemma 2.3, we get

$$
\begin{aligned}
\lambda(1-\lambda)\left\|S_{n, m} x_{n}-f\right\| & =\|\lambda x+(1-\lambda) y\| \\
& \leq \lambda(1-\lambda)(r+d)\left[1-2 \lambda(1-\lambda) \delta\left(\frac{\varepsilon}{\lambda(1-\lambda)(r+d)}\right)\right] \\
& \leq \lambda(1-\lambda)(r+d)\left[1-2 \lambda(1-\lambda) \delta\left(\frac{\varepsilon}{r+d}\right)\right]
\end{aligned}
$$


and then by (3.10),

$$
\begin{aligned}
r-d & \leq\left\|x_{n+m}-f\right\|=\left\|S_{n, m} x_{n}-f\right\| \\
& \leq(r+d)\left[1-2 \lambda(1-\lambda) \delta\left(\frac{\varepsilon}{r+d}\right)\right],
\end{aligned}
$$

which contradicts (3.9). Thus we can conclude that for all $n>n_{0}$,

$$
\left\|S_{n, m}\left[\lambda x_{n}+(1-\lambda) f\right]-\left[\lambda S_{n, m} x_{n}+(1-\lambda) f\right]\right\| \leq \varepsilon, \quad \forall m \in N .
$$

Hence by (3.11), for all $n>n_{0}$,

$$
\begin{aligned}
& \left\|\lambda x_{n+m}+(1-\lambda) f-g\right\| \\
= & \left\|\lambda S_{n, m} x_{n}+(1-\lambda) f-g\right\| \\
\leq & \left\|\left[\lambda S_{n, m} x_{n}+(1-\lambda) f\right]-S_{n, m}\left[\lambda x_{n}+(1-\lambda) f\right]\right\|+\left\|S_{n, m}\left[\lambda x_{n}+(1-\lambda) f\right]-g\right\| \\
\leq & \varepsilon+\left\|\lambda x_{n}+(1-\lambda) f-g\right\|+\left(w_{n+m-1}+\cdots+w_{n+1}+w_{n}\right) \\
\leq & 2 \varepsilon+\left\|\lambda x_{n}+(1-\lambda) f-g\right\| .
\end{aligned}
$$

For any fixed $n>n_{0}$, we can take the limsup for $m$ and obtain

$$
\limsup _{m \rightarrow+\infty}\left\|\lambda x_{m}+(1-\lambda) f-g\right\| \leq\left\|\lambda x_{n}+(1-\lambda) f-g\right\|+2 \varepsilon .
$$

Hence

$$
\limsup _{m \rightarrow+\infty}\left\|\lambda x_{m}+(1-\lambda) f-g\right\| \leq \liminf _{n \rightarrow+\infty}\left\|\lambda x_{n}+(1-\lambda) f-g\right\|+2 \varepsilon .
$$

Since $\varepsilon>0$ is arbitrary, this implies that

$$
h(\lambda)=\lim _{n \rightarrow+\infty}\left\|\lambda x_{n}+(1-\lambda) f-g\right\|
$$

exists. This completes the proof.

Remark 3.1 If the mappings are asymptotically nonexpansive, we can use Bruck's Lemma [23] to prove Lemma 3.3. While Bruck's Lemma is not valid for non-Lipschitzian mappings, we must introduce new technique to establish a similar inequality. In [20], Chidume et al. also proved that $\lim _{n \rightarrow+\infty} \| \lambda x_{n}+(1-\lambda) f-g$ lexists (Lemma 3.12 in [20]). As we have seen, our proof is completely different from theirs in [20].

Lemma 3.4 If $f \in \omega_{\omega}\left(\left\{x_{n}\right\}\right)$ and $\lim _{n \rightarrow+\infty}\left\|\lambda x_{n}+(1-\lambda) f-g\right\|_{\text {exists, then }}$

$$
h(\lambda)=\lim _{n \rightarrow+\infty}\left\|\lambda x_{n}+(1-\lambda) f-g\right\| \leq\|f-g\| .
$$

Proof. For any $\varepsilon>0$, there exists $n_{0}$ such that for all $n \geq n_{0}$,

$$
\left\|\lambda x_{n}+(1-\lambda) f-g\right\| \leq h(\lambda)+\varepsilon .
$$

Then for all $n \geq n_{0}$,

$$
\left\langle\lambda x_{n}+(1-\lambda) f-g_{\prime} J(f-g)\right\rangle \leq\|f-g\|(h(\lambda)+\varepsilon) .
$$

Since $f \in \omega_{\omega}\left(\left\{x_{n}\right\}\right)$, there exists a subsequence $\left\{x_{n_{i}}\right\} \subset\left\{x_{n}\right\}$ with $x_{n_{i}} \rightarrow f$. Hence $f \in \bar{c} o\left\{x_{n_{i}}, i \geq n_{0}\right\}$ and

$$
\{\lambda f+(1-\lambda) f-g, J(f-g)\} \leq\|f-g\|(h(\lambda)+\varepsilon),
$$

i.e., $\|f-g\|^{2} \leq\|f-g\|(h(\lambda)+\varepsilon)$. Therefore $\|f-g\| \leq h(\lambda)$. This completes the proof. Now we can prove the weak convergence theorem of the iterative sequence (3.1). 
Theorem 3.1 Let $C$ be a nonempty closed convex subset of uniformly convex Banach space $X$ which satisfies the Opial's condition or whose dual $X^{*}$ has the Kadec-Klee property. Let $P: X \rightarrow C$ be a nonexpansive retraction from $X$ onto $C$. Let $T_{1}, T_{2}, T_{3}: C \rightarrow X$ be three asymptotically nonexpansive in the intermediate sense nonself mappings with $F$ $\neq \varnothing$ and the nonnegative sequence $\left\{r_{n}\right\}$ satisfy $\sum_{n=1}^{+\infty} r_{n}<+\infty$. Let $\left\{x_{n}\right\}$ be defined by: $x_{1} \in$ $C$ and

$$
\begin{aligned}
& x_{n+1}=P\left[\alpha_{n}^{(1)} z_{n}+\left(1-\alpha_{n}^{(1)}\right) T_{1}\left(P T_{1}\right)^{n-1} z_{n}\right] ; \\
& z_{n}=P\left[\alpha_{n}^{(2)} y_{n}+\left(1-\alpha_{n}^{(2)}\right) T_{2}\left(P T_{2}\right)^{n-1} y_{n}\right] \\
& y_{n}=P\left[\alpha_{n}^{(3)} x_{n}+\left(1-\alpha_{n}^{(3)}\right) T_{3}\left(P T_{3}\right)^{n-1} x_{n}\right] .
\end{aligned}
$$

where $\left\{\alpha_{n}^{(i)}\right\}$ is in $[0,1]$ with $0<p \leq \alpha_{n}^{(i)} \leq q<1, i=1,2,3$. Then $\left\{x_{n}\right\},\left\{y_{n}\right\}$, and $\left\{z_{n}\right\}$ converge weakly to a common fixed point of $\left\{T_{i}\right\}_{i=1}^{3}$.

Proof. It suffices to show that $\left\{x_{n}\right\}$ converges weakly to a common fixed point of $\left\{T_{i}\right\}_{i=1}^{3}$. To this aim, we only need to prove that the set $\omega_{\omega}\left(\left\{x_{n}\right\}\right)$ is singleton. Since $X$ is reflexive and $C$ is bounded, we obtain $\omega_{\omega}\left(\left\{x_{n}\right\}\right) \neq \varnothing$. Assume that $f, g \in \omega_{\omega}\left(\left\{x_{n}\right\}\right)$, then there exist two subsequences $\left\{x_{n_{i}}\right\}$ and $\left\{x_{n_{j}}\right\}$ in $\left\{x_{n}\right\}$ such that $x_{n_{i}} \rightarrow$ fand $x_{n_{j}} \rightarrow g$. In the following, we shall show $f=g$. By Lemmas 2.5 and 3.2, $f, g \in F$. On one hand, if $X$ satisfies the Opial's condition and $f \neq g$, then by the Lemma 3.1, we get

$$
\begin{aligned}
r & =\lim _{n \rightarrow+\infty}\left\|x_{n}-f\right\|=\lim _{i \rightarrow+\infty}\left\|x_{n_{i}}-f\right\| \\
& <\lim _{i \rightarrow+\infty}\left\|x_{n_{i}}-g\right\|=\lim _{n \rightarrow+\infty}\left\|x_{n}-g\right\|=\lim _{j \rightarrow+\infty}\left\|x_{n_{j}}-g\right\| \\
& <\lim _{j \rightarrow+\infty}\left\|x_{n_{j}}-f\right\|=\lim _{n \rightarrow+\infty}\left\|x_{n}-f\right\|=r .
\end{aligned}
$$

This contraction implies $f=g$. On the other hand, if $X^{*}$ has Kadec-Klee property, then from Lemmas 2.4, 3.3, and 3.4, we have

$$
\begin{aligned}
& \left\|\lambda x_{n}+(1-\lambda) f-g\right\|^{2} \\
\leq & \|f-g\|^{2}+2 \lambda\left\langle x_{n}-f, J\left(\lambda x_{n}+(1-\lambda) f-g\right)\right\rangle
\end{aligned}
$$

and for all $\lambda \in[0,1]$,

$$
\liminf _{n \rightarrow+\infty}\left\langle x_{n}-f, J\left(\lambda x_{n}+(1-\lambda) f-g\right)\right\rangle \geq 0 .
$$

Hence

$$
\liminf _{j \rightarrow+\infty}\left\langle x_{n_{j}}-f, J\left(\lambda x_{n_{j}}+(1-\lambda) f-g\right)\right\rangle \geq 0 .
$$

Thus for arbitrary $k \in N$, there exists $j_{k} \geq k,\left\{j_{k}\right\} \uparrow$, such that

$$
\left\langle x_{n_{j_{k}}}-f, J\left(\frac{1}{k} x_{n_{j_{k}}}+\left(1-\frac{1}{k}\right) f-g\right)\right\rangle \geq-\frac{1}{k} .
$$

Obviously $x_{n_{j_{k}}} \rightarrow g$. Put

$$
j_{k}=J\left(\frac{1}{k} x_{n_{j_{k}}}+\left(1-\frac{1}{k}\right) f-g\right),
$$

then we may assume that, without loss of generality, $j_{k}$ is weakly convergent to some 
point $j \in X^{*}$. Therefore $\|j\| \leq \liminf _{k \rightarrow+\infty}\left\|j_{k}\right\|=\|f-g\|$. Noting

$$
\left\langle f-g, j_{k}\right\rangle=\left\|\frac{1}{k} x_{n_{j_{k}}}+\left(1-\frac{1}{k}\right) f-g\right\|^{2}-\frac{1}{k}\left\langle x_{n_{j_{k}}}-f, j_{k}\right\rangle
$$

and passing the limit for $k$, we have $\langle f-g, j\rangle=\|f-g\|^{2}$. Hence $\|j\| \geq\|f-g\|$ and

$$
\langle f-g, j\rangle=\|f-g\|^{2}=\|j\|^{2},
$$

which means $j=J(f-g)$. Thus we can conclude $j_{k} \rightarrow j$ and $\left\|j_{k}\right\| \rightarrow|| f-g\|=\| j \|$. Since $X^{*}$ has Kadec-Klee property, we have $j_{k} \rightarrow j$. Taking the limit in (3.12), we get $\langle g$ $-f, j\rangle \geq 0$, i.e., $\|f-g\|^{2} \leq 0$, which implies $f=g$. This completes the proof.

Remark 3.2 Theorem 3.1 extends the main results in $[4,16,18,20]$ to the case of asymptotically nonexpansive in the intermediate sense mappings and it seems to be new even in the case that the space has a Fréchet differentiable norm.

In the following, we shall give a nonself mapping which is asymptotically nonexpansive in the intermediate sense but not asymptotically nonexpansive.

Example 3.1 Let $\Delta$ be the Cantor ternary set. Define the Cantor ternary function

$$
\tau(x)= \begin{cases}\sum_{n=1}^{+\infty} \frac{b_{n}}{2^{n}} & x=\sum_{n=1}^{+\infty} \frac{2 b_{n}}{3^{n}} \in \Delta,\left(b_{n}=0,1\right) \\ \sup \{\tau(y), y \leq x, y \in \Delta\} & x \in[0,1] \backslash \Delta\end{cases}
$$

then $\tau:[0,1] \rightarrow[0,1]$ is a continuous and increasing but not absolutely continuous function with $\tau(0)=0, \tau\left(\frac{1}{2}\right)=\frac{1}{2}$ (see [27]). Since a Lipschitzian function is absolutely continuous, $\tau$ is non-Lipschitzian. Define $\phi: R \rightarrow R$ by

$$
\varphi(x)= \begin{cases}0 & x<0 \text { or } x>1 \\ \frac{x}{2} & 0 \leq x \leq \frac{1}{2} \\ \frac{1}{2} \tau(1-x) & \frac{1}{2}<x \leq 1\end{cases}
$$

It is easy to see that $\phi$ is continuous and for all $x, y \in R,|\varphi(x)-\varphi(y)| \leq \frac{1}{2}$. It also can be verified that the $n$-fold composition mapping $\phi^{n}$ is defined by

$$
\varphi^{n}(x)= \begin{cases}0 & x<0 \text { or } x>1 \\ \frac{x}{2^{n}} & 0 \leq x \leq \frac{1}{2} \\ \frac{1}{2^{n}} \tau(1-x) & \frac{1}{2}<x \leq 1\end{cases}
$$

Since $\tau$ is non-Lipschitzian, so is $\phi^{n}$ and for all $x, y \in R$,

$$
\left|\varphi^{n}(x)-\varphi^{n}(y)\right| \leq \frac{1}{2^{n}} .
$$

Taking $X=R^{2}$ with the norm $\|(x, y)\|=\sqrt{x^{2}+y^{2}},(x, y) \in X$ and $C=R \times\{0\}$, we define the nonself mapping $T: C \rightarrow X$ by

$$
T(x, 0)=(\varphi(x), x), \quad(x, 0) \in C,
$$

then $T$ is continuous and $(0,0)$ is a fixed point of $T$. Define $P: X \rightarrow C$ by

$$
P(x, y)=(x, 0), \quad(x, y) \in X,
$$

then $P$ is a nonexpansive retraction from $X$ onto $C$. Hence for all $(x, 0),(y, 0) \in C$,

$$
T(P T)^{n-1}(x, 0)=\left(\varphi^{n}(x), \varphi^{n-1}(x)\right),
$$


which means $T(P T)^{n-1}$ is is non-Lipschitzian and

$$
\begin{aligned}
& \left\|T(P T)^{n-1}(x, 0)-T(P T)^{n-1}(y, 0)\right\| \\
= & \left\|\left(\varphi^{n}(x), \varphi^{n-1}(x)\right)-\left(\varphi^{n}(y), \varphi^{n-1}(y)\right)\right\| \\
= & \sqrt{\left(\varphi^{n}(x)-\varphi^{n}(y)\right)^{2}+\left(\varphi^{n-1}(x)-\varphi^{n-1}(y)\right)^{2}} \\
\leq & \left|\varphi^{n}(x)-\varphi^{n}(y)\right|+\left|\varphi^{n-1}(x)-\varphi^{n-1}(y)\right| \\
\leq & \|(x, 0)-(y, 0)\|+\frac{3}{2^{n}} .
\end{aligned}
$$

Therefore, we can conclude that $T$ is asymptotically nonexpansive in the intermediate sense but not an asymptotically nonexpansive.

If $T_{1}, T_{2}$, and $T_{3}$ are nonexpansive, we can prove the following theorem.

Theorem 3.2 Let $C$ be a nonempty closed convex subset of uniformly convex Banach space $X$ which satisfies the Opial's condition or whose dual has the Kadec-Klee property. Let $P: X \rightarrow C$ be a nonexpansive retraction from $X$ onto $C$. Let $T_{1}, T_{2}, T_{3}: C \rightarrow$ $X$ be three nonexpansive nonself mappings and $\left\{x_{n}\right\}$ be defined by: $x_{1} \in C$ and

$$
\begin{aligned}
& x_{n+1}=P\left[\alpha_{n}^{(1)} z_{n}+\left(1-\alpha_{n}^{(1)}\right) T_{1} z_{n}\right] ; \\
& z_{n}=P\left[\alpha_{n}^{(2)} y_{n}+\left(1-\alpha_{n}^{(2)}\right) T_{2} y_{n}\right] ; \\
& y_{n}=P\left[\alpha_{n}^{(3)} x_{n}+\left(1-\alpha_{n}^{(3)}\right) T_{3} x_{n}\right] .
\end{aligned}
$$

where $\left\{\alpha_{n}^{(i)}\right\}$ is in $0[1]$ with $0<p \leq \alpha_{n}^{(i)} \leq q<1, i=1,2$, 3. Then $\left\{x_{n}\right\}$, $\left\{y_{n}\right\}$ and $\left\{z_{n}\right\}$ converge weakly to a common fixed point of $\left\{T_{i}\right\}_{i=1}^{3}$.

Remark 3.3 We would like to remark that if the so-called error terms are added in our recursion formula and are assumed to be bounded, then the results of this article still hold. Thus we can get the main results in [19].

\section{Acknowledgements}

This research is supported by the National Natural Science Foundation of China (10971182), the Natural Science Foundation of Jiangsu Province (BK2009179 and BK2010309), the Tianyuan Youth Foundation (11026115), the Jiangsu Government Scholarship for Overseas Studies, the Natural Science Foundation of Jiangsu Education Committee (09KJB110010 and 10KJB110012) and the Natural Science Foundation of Yangzhou University.

\section{Authors' contributions}

All authors read and approved the final manuscript.

\section{Competing interests}

The authors declare that they have no competing interests.

Received: 20 August 2011 Accepted: 30 December 2011 Published: 30 December 2011

\section{References}

1. Mann, WR: Mean value methods in iteration. Proc Amer Math Soc. 4(3), 506-510 (1953). doi:10.1090/S0002-9939-19530054846-3

2. Ishikawa, S: Fixed points and iteration of a nonexpansive mapping in a Banach space. Proc Amer Math Soc. 59(1), 65-71 (1967)

3. Groetsch, CW: A note on segmenting Mann iterates. J Math Anal Appl. 40, 369-372 (1972). doi:10.1016/0022-247X(72) 90056-X

4. Schu, J: Weak and strong convergence to fixed points of asymptotically non-expansive mappings. Bull Austral Math Soc. 43(1), 153-159 (1991). doi:10.1017/S0004972700028884

5. Tan, KK, Xu, HK: Approximating fixed points of nonexpansive mappings by the Ishikawa iteration process. J Math Anal Appl. 178, 301-308 (1993). doi:10.1006/jmaa.1993.1309

6. Noor, MA: New approximation schemes for general variational inequalities. J Math Anal Appl. 251, $217-229$ (2000). doi:10.1006/jmaa.2000.7042

7. Xu, B, Noor, MA: Fixed-point iterations for asymptotically nonexpansive mappings in Banach spaces. J Math Anal Appl. 267(2), 444-453 (2002). doi:10.1006/jmaa.2001.7649 
8. Cho, Y, Zhou, H, Guo, G: Weak and strong convergence theorems for three-step iterations with errors for asymptotically nonexpansive mappings. Comput Math Appl. 47(4-5), 707-717 (2004). doi:10.1016/50898-1221(04)90058-2

9. Suantai, S: Weak and strong convergence criteria of Noor iterations for asymptotically nonexpansive mappings. J Math Anal Appl. 311, 506-517 (2005). doi:10.1016/j.jmaa.2005.03.002

10. Shahzad, N, Udomene, A: Fixed point solutions of variational inequalities for asymptotically nonexpansive mappings in Banach spaces. Nonlinear Anal: Theory Methods Appl. 64(3), 558-567 (2006). doi:10.1016/j.na.2005.03.114

11. Shahzad, N, Udomene, A: Approximating common fixed points of two asymptotically quasi-nonexpansive mappings in Banach spaces. Fixed Point Theory and Applications 2006, 10 (2006). Article ID 18909

12. Plubtieng, S, Wangkeeree, R, Punpaeng, R: On the convergence of modified Noor iterations with errors for asymptotically nonexpansive mappings. J Math Anal Appl. 322, 1018-1029 (2006). doi:10.1016/j.jmaa.2005.09.078

13. Cai, G, Hu, C: On strong convergence by the hybrid method for equilibrium and fixed point problems for an inifnite family of asymptotically nonexpansive mappings. Fixed Point Theory and Applications 2009, 20 (2009). Article ID 798319

14. Chidume, $\mathrm{CE}$, Zegeye, $\mathrm{H}$, Shahzad, $\mathrm{N}$ : Convergence theorems for a common fixed point of finite family of nonself nonexpansive mappings. Fixed Point Theory Appl. 2005, 1-9 (2005)

15. Shahzad, N: Approximating fixed points of non-self nonexpansive mappings in Banach spaces. Nonlinear Anal: Theory, Methods Appl. 61, 1031-1039 (2005). doi:10.1016/j.na.2005.01.092

16. Chidume, CE, Ofoedu, EU, Zegeye, H: Strong and weak convergence theorems for asymptotically nonexpansive mappings. J Math Anal Appl. 280, 364-374 (2003). doi:10.1016/50022-247X(03)00061-1

17. Wang, C, Zhu, J: Convergence theorems for common fixed points of nonself asymptotically quasi-non-expansive mappings. Fixed Point Theory and Applications 2008, 11 (2008). Article ID 428241

18. Thianwan, $\mathrm{S}$ : Common fixed points of new iterations for two asymptotically nonexpansive nonself-mappings in a Banach space. J Comput Appl Math. 224, 688-695 (2009). doi:10.1016/j.cam.2008.05.051

19. Huang, QL, Hu, Y, Gao, SY: Convergence theorems for three-step iterations with errors of asymptotically nonexpansive nonself mappings in Banach spaces. Math Appl. 24, 540-547 (2011)

20. Chidume, CE, Shahzad, N, Zegeye, H: Convergence theorems for mappings which are asymptotically nonexpansive in the intermediate sense. Numer Funct Anal Optimiz. 25, 239-257 (2004)

21. Kim, GE, Kim, TH: Mann and Ishikawa iterations with errors for non-Lipschitzian mappings in Banach spaces. Comput Math Appl. 42, 1565-1570 (2001). doi:10.1016/S0898-1221(01)00262-0

22. Plubtieng, S, Wangkeeree, R: Strong convergence theorems for three-step iterations with errors for non-Lipschitzian nonself-mappings in Banach Spaces. Comput Math Appl. 51, 1093-1102 (2006). doi:10.1016/j.camwa.2005.08.035

23. Bruck, RE: A simple proof of the mean ergodic theorem for nonlinear contractions in Banach spaces. Israel J Math. 32, 107-116 (1979). doi:10.1007/BF02764907

24. Kaczor, W: A nonstandard proof of a generalized demiclosedness principle. pp. 43-50. Annales Universitatis Mariae Curie-Sklodowska Lublin-Polonia LIX (2005)

25. Kaczor, W: Weak convergence of almost orbits of asymptotically nonexpansive commutative semigroups. J Math Anal Appl. 272, 565-574 (2002). doi:10.1016/50022-247X(02)00175-0

26. Chang, SS: Some problems and results in the study of nonlinear analysis. Nonlinear Anal: Theory Methods Appl. 30, 4197-4208 (1997). doi:10.1016/S0362-546X(97)00388-X

27. Royden, HL: Real Analysis, 3rd edn.Pearson Education (2004)

doi:10.1186/1687-1812-2011-106

Cite this article as: Zhu et al:: Weak convergence theorem for the three-step iterations of non-Lipschitzian

nonself mappings in Banach spaces. Fixed Point Theory and Applications 2011 2011:106.

\section{Submit your manuscript to a SpringerOpen ${ }^{\circ}$ journal and benefit from:}

Convenient online submission

- Rigorous peer review

- Immediate publication on acceptance

- Open access: articles freely available online

- High visibility within the field

- Retaining the copyright to your article

Submit your next manuscript at $\gg$ springeropen.com 